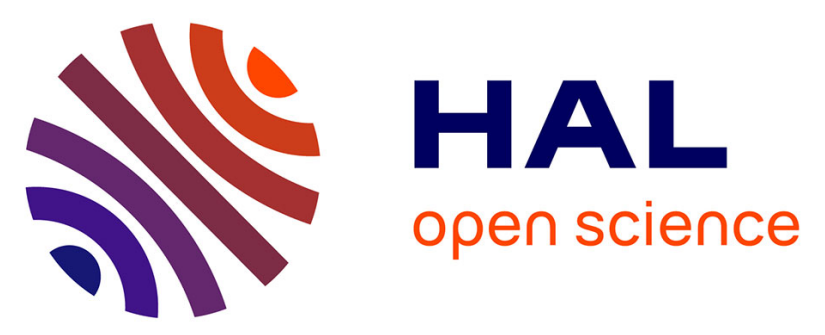

\title{
Effect of structural distortions on the magnetism of doped spin-Peierls CuGeO3
}

Virginie Simonet, Beatrice Grenier, Françoise Villain, Anne-Marie Flank, Guy Dhalenne, Alexandre Revcolevschi, Jean-Pierre Renard

\section{- To cite this version:}

Virginie Simonet, Beatrice Grenier, Françoise Villain, Anne-Marie Flank, Guy Dhalenne, et al.. Effect of structural distortions on the magnetism of doped spin-Peierls CuGeO3. The European Physical Journal B: Condensed Matter and Complex Systems, 2006, 53, pp.155. 10.1140/epjb/e2006-00357-8 . hal-00090850v2

\section{HAL Id: hal-00090850 \\ https://hal.science/hal-00090850v2}

Submitted on 14 Aug 2008

HAL is a multi-disciplinary open access archive for the deposit and dissemination of scientific research documents, whether they are published or not. The documents may come from teaching and research institutions in France or abroad, or from public or private research centers.
L'archive ouverte pluridisciplinaire HAL, est destinée au dépôt et à la diffusion de documents scientifiques de niveau recherche, publiés ou non, émanant des établissements d'enseignement et de recherche français ou étrangers, des laboratoires publics ou privés. 


\title{
Effect of structural distortions on the magnetism of doped spin-Peierls $\mathrm{CuGeO}_{3}$
}

\author{
V. Simonet ${ }^{1}$, B. Grenier ${ }^{2,3}$, F. Villain ${ }^{4}$, A.-M. Flank ${ }^{5}$, G. Dhalenne ${ }^{6}$, A. Revcolevschi ${ }^{6}$, and J.-P. Renard ${ }^{7}$ \\ 1 Laboratoire Louis Néel, UPR 5051, B.P. 166, F-38042 Grenoble Cedex 9, France \\ 2 CEA-Grenoble, DRFMC / SPSMS / MDN, F-38054 Grenoble Cedex 9, France \\ 3 Université Joseph Fourier, B.P. 53, F-38041 Grenoble Cedex 9, France \\ ${ }^{4}$ Laboratoire de Chimie Inorganique et Matériaux Moléculaires, UMR 7071, UPMC, 4 place Jussieu, F-75252 Paris Cedex 05, \\ France \\ 5 SOLEIL, L'Orme des Merisiers, Saint-Aubin - BP 48, F-91192 Gif-sur-Yvette Cedex, France \\ 6 Laboratoire de Physico-Chimie de l'Etat Solide, UMR 8648, Université Paris-Sud, Bât. 414, F-91405 Orsay Cedex, France \\ 7 Institut d'électronique Fondamentale, UMR 8622, Bât. 220, Université Paris-Sud, F-91405 Orsay Cedex, France
}

Received: date / Revised version: date

\begin{abstract}
The chemical selectivity and great sensitivity of the Extended X-ray Absorption Spectroscopy technique allowed the determination, in the paramagnetic phase, of the structural distortions induced by doping in the spin-Peierls $\mathrm{CuGeO}_{3}$ compound. The distorted environments were analyzed as a function of concentration, magnetic nature of impurity and the substitution site ( $\mathrm{Ni}, \mathrm{Mn}$ and $\mathrm{Zn}$ impurities on the $\mathrm{Cu}$ site, Si impurity on the Ge site). This has led to estimate the variation of the angles and pair distances, and hence to evaluate the magnetic coupling along the $\mathrm{Cu}$ chains in the vicinity of the impurities. The antiferromagnetic interaction between $\mathrm{Cu}$ first neighbors in the pure sample is found to be weakened around $\mathrm{Ni}$, almost cancelled in the case of $\mathrm{Mn}$ doping, and even to change sign, producing a ferromagnetic coupling for Si doping. More generally, the structural distortions on a local scale are shown to be key parameters for the understanding of the magnetic properties of doped spin-Peierls compounds.
\end{abstract}

PACS. 61.10.Ht X-ray absorption spectroscopy: EXAFS, NEXAFS, XANES, etc. - 61.72.-y Defects and impurities in crystals; microstructure - 75.10.Pq Spin chain models

\section{Introduction}

The first inorganic spin-Peierls (SP) compound, $\mathrm{CuGeO}_{3}$, [1] has already been extensively studied for its original magnetic properties. The possibility of substituting the cationic sites, $\mathrm{Cu}$ or $\mathrm{Ge}$, by different magnetic or non-magnetic elements, has brought new information and rose new questions.

The structure of $\mathrm{CuGeO}_{3}$ can be described as a stacking of $\mathrm{CuO}_{6}$ octahedra and $\mathrm{GeO}_{4}$ tetrahedra along the $c$ direction of the structure. Each $\mathrm{O}$ belongs to both a Ge tetrahedron and a $\mathrm{Cu}$ octahedron. The stacking of distorted octahedra (elongated along the direction of the apical $\mathrm{O}(1)$ and with a planar distorted square of basal $\mathrm{O}(2)$ in the perpendicular direction) results in chains of magnetic $\mathrm{Cu}^{2+}$ ions located at the center of edge sharing squares of $\mathrm{O}(2)$ running along the $c$ axis (Fig. 1). At room temperature, this compound then consists of uniform chains of spins $S=1 / 2$, interacting by superexchange through $\mathrm{O}(2)$ atoms leading to an antiferromagnetic (AF) Heisenberg-type coupling (Fig. 11). A magnetoelastic instability produces a dimerization of the chains below a critical temperature $T_{S P}$ of $14.25 \mathrm{~K}$. This leads to a sin-

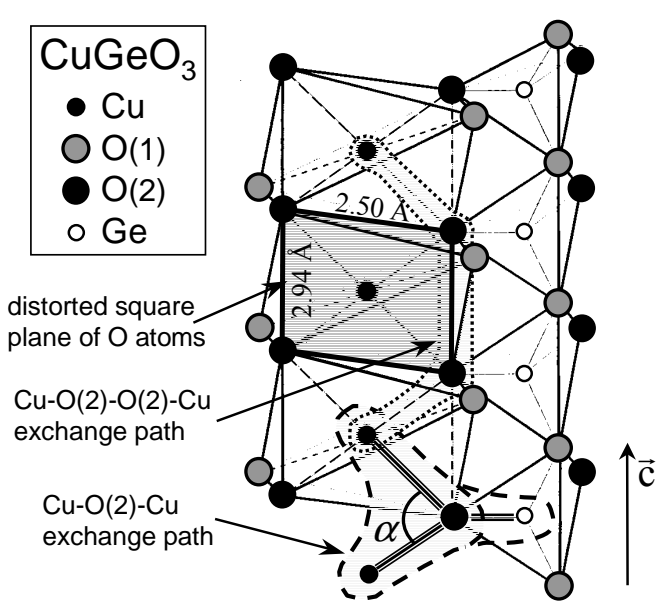

Fig. 1. Schematic structure of $\mathrm{CuGeO}_{3}$

glet (non-magnetic) ground state separated from the first excited triplet state $(S=1)$ by an energy gap of $2 \mathrm{meV}$. Below $T_{S P}$, the magnetic susceptibility drops to zero with 
a temperature activated behavior, and diffraction experiments reveal new superlattice peaks [2].

Doping, either with $\mathrm{Zn}, \mathrm{Ni}, \mathrm{Mg}$ or $\mathrm{Mn}$ on the $\mathrm{Cu}$ site 3 . 4,5] or with $\mathrm{Si}$ on the Ge site [6,6], results into a decrease of $T_{S P}$ and, at a lower temperature, the Néel temperature $T_{N}$, into a transition to an AF long-range ordering (LRO). Some of these impurities are magnetic, like $\mathrm{Ni}$ with spin 1 and $\mathrm{Mn}$ with spin 5/2 $|8|$, while the others, $\mathrm{Zn}, \mathrm{Mg}$ and $\mathrm{Si}$, are non magnetic. They also have very different ionic radii whose consequence on the magnetic properties will be discussed in Sec. IV and V. A systematic study of the magnetic properties of single crystals with various doping levels $\left(\mathrm{CuGe}_{1-x} \mathrm{Si}_{x} \mathrm{O}_{3}\right.$ with $0<x \leq 0.08$, and $\mathrm{Cu}_{1-y} M_{y} \mathrm{GeO}_{3}$ with $M=\mathrm{Zn}, \mathrm{Mg}, \mathrm{Ni}$ and $0<y \leq 0.1$ ), all synthesized and characterized in the same way, has allowed establishing a quasi-universal phase diagram for the spin-Peierls transition [5]. The variation of $T_{S P}$ versus concentration was found identical for all impurities on the $\mathrm{Cu}$ site, including $\mathrm{Mn}$, which has been studied since Ref. [5] (for $0<y \leq 0.02$ ), and for Si impurities applying a scaling factor $y \approx 3 x$. This is illustrated in Fig. 2 of Ref. [5] and in the alternative representation of the present Fig. 2 2 where no scaling factor $y \approx 3 x$ is applied in view of the discussion of Sec. V.B. Surprisingly, doping outside the spin chains is thus about three times more efficient in destroying the spin-Peierls phase than doping within the chains ${ }^{1}$. In contrast, $T_{N}$ varies with the type of impurity: it is higher for $\mathrm{Mn}$ and $\mathrm{Si}$-doping and slightly lower for Ni-doping, in comparison with the value, found identical, for the non-magnetic impurities $\mathrm{Mg}$ and $\mathrm{Zn}$. A difference between Ge site and $\mathrm{Cu}$ site doping is observed concerning the $T_{N}(y, x)$ transition line which is continuous in the first case and presents a kink between a dimerized AF phase at low doping and a uniform AF phase at larger doping in the second case $[12$. This behaviour is characteristic of a second-order and a first-order phase transition, respectively. Another particularity concerns the easy axis of magnetization in the AF phase: it is the $a$-axis for $\mathrm{Ni}$ doping and the $c$-axis for all other substitutions, which can be explained by the single-ion anisotropy of $\mathrm{Ni}^{2+}$ in a distorted octahedral environment [13].

These results must be considered in the light of the different couplings along the $\mathrm{Cu}$ chains. The magnetic properties are mainly driven by two kinds of AF couplings: A superexchange coupling $\mathrm{Cu}-\mathrm{O}(2)-\mathrm{Cu}$ between first $\mathrm{Cu}$

\footnotetext{
1 Note that, while for $\mathrm{Ni}, \mathrm{Zn}$ and $\mathrm{Mg}$, the results of Grenier et al. 5] are in rather good agreement with the work of other groups on crystals of different origin, a large spread of results exists concerning Si-doping. The slope of $T_{S P}(x)$ from the works of Katano et al. 9] and Hiroi et al. [10] is smaller than that found by Grenier et al. while it is similar in the work of Wang et al. 11], leading to the scaling factors $y=x, y=1.5 x$ and $y \sim 2-3 x$ respectively. However, we are more confident in the results of Grenier et al. concerning the Si-doping data since the weaker effect measured by other groups suggests that not all $\mathrm{Si}$ ions enter the $\mathrm{CuGeO}_{3}$ structure and/or part of them segregates (as was already observed in highly doped samples). This leads to overestimate $x$ and thus results in an apparent smaller scaling factor between $y$ and $x$.
}

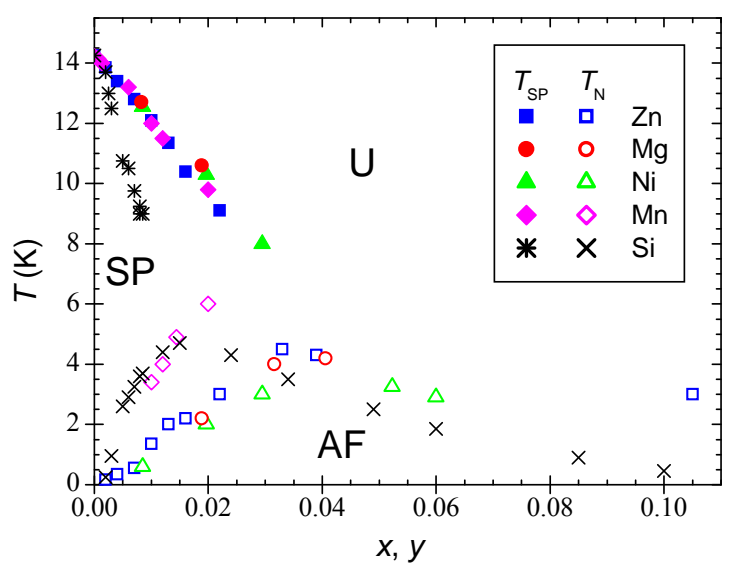

Fig. 2. (Temperature - concentration) magnetic phase diagram for $\mathrm{CuGe}_{1-x} \mathrm{Si}_{x} \mathrm{O}_{3}$ and $\mathrm{Cu}_{1-y} M_{y} \mathrm{GeO}_{3}$, with $M=\mathrm{Zn}, \mathrm{Mg}, \mathrm{Ni}$, and $\mathrm{Mn}$.

neighbors with an AF exchange constant $J_{1}=120-180$ $\mathrm{K}$ and a frustrating AF coupling between second neighbors $J_{2}=0.24-0.36 J_{1}$ via the $\mathrm{Cu}-\mathrm{O}(2)-\mathrm{O}(2)-\mathrm{Cu}$ path [14, 15] (Fig. 11). The inter-chain couplings are one order and two orders of magnitude smaller along the $b$ and $a$ axes, respectively 15. These inter-chain couplings induce a coherence between the phases of the distortion waves on different chains. In $\mathrm{CuGeO}_{3}$, the dimerization pattern is out of phase for adjacent chains in the $b$ and $a$ directions. The strength and nature of the magnetic interactions are strongly dependent on the $\mathrm{Cu}-\mathrm{O}(2)$ and $\mathrm{Ge}-$ $\mathrm{O}(2)$ distances and even more drastically on the $\mathrm{Cu}-\mathrm{O}(2)$ $\mathrm{Cu}$ superexchange angle (denoted $\alpha$ ). The GoodenoughKanamori-Anderson rules 16 state that the exchange is ferromagnetic (FM) for an angle of $90^{\circ}$. The deviation of the $\mathrm{Cu}-\mathrm{O}(2)-\mathrm{Cu}$ angle from this value $\left(\alpha=99^{\circ}\right.$ in $\left.\mathrm{CuGeO}_{3}\right)$ will drive the exchange towards antiferromagnetism [17]. However, Geertsma and Khomskii pointed out that calculations taking into account this sole effect still yield a slightly ferromagnetic exchange coupling |18: The hybridization of Ge orbitals with the $2 p_{y}$ ones of the $\mathrm{O}(2)$, which destroys the equivalence of $2 p_{x}$ and $2 p_{y} \mathrm{O}(2)$ orbitals, must indeed be taken into account to obtain, for $J_{1}$, an AF exchange with a reasonable value of $135 \mathrm{~K}$.

The aim of the present work is to understand the effect of doping the $\mathrm{Cu}$ chains, with magnetic and non-magnetic impurities, and out of the chains in the case of Si-doping, by measuring the induced structural distortions of doped $\mathrm{CuGeO}_{3}$. These distortions should strongly influence the magnetic properties, in particular via the variation of the superexchange angle $\alpha$ and thus of the related magnetic coupling $J_{1}$. The EXAFS (Extended X-ray Absorption Fine Structure) spectroscopy technique was chosen for this purpose as a local order probe, with chemical sensitivity, and for its possibility to measure very diluted systems. For the first time with this technique, the influence of $\mathrm{Ni}, \mathrm{Mn}$, $\mathrm{Zn}$ and Si-doping was investigated in the paramagnetic 

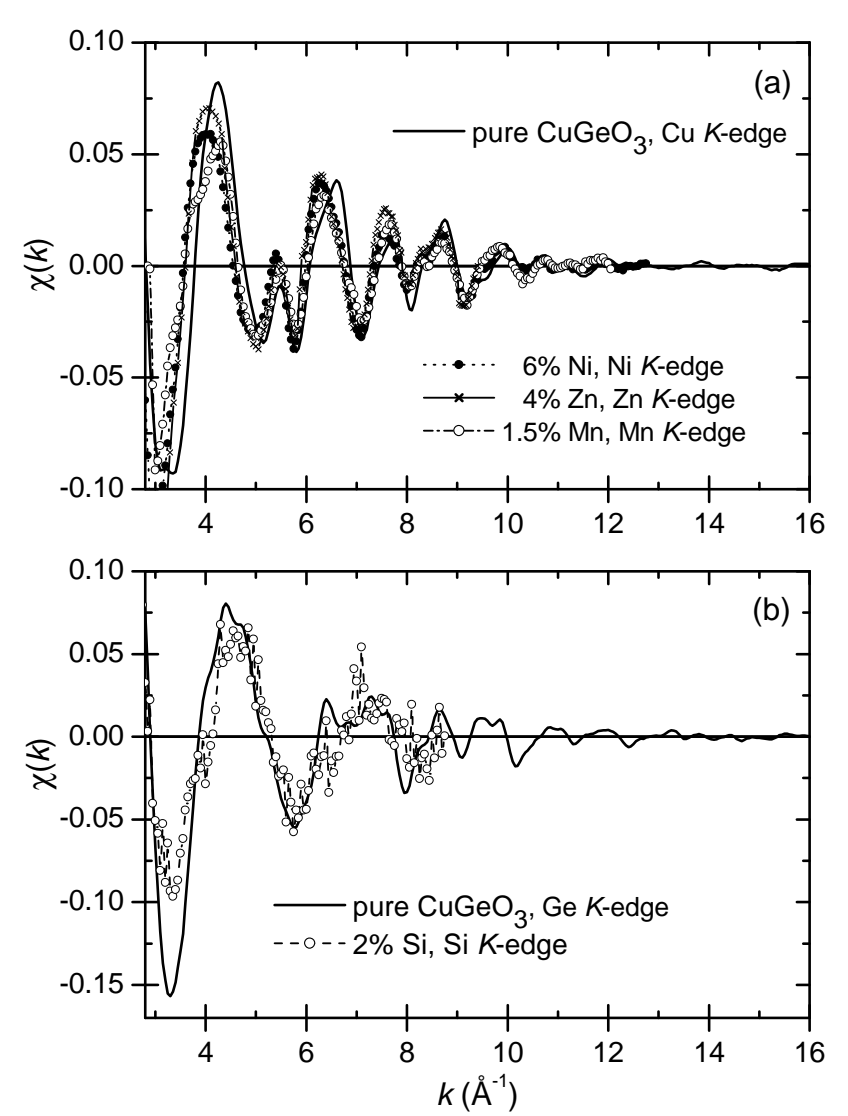

Fig. 3. Comparison of the measured $\chi(k)$ functions (a) for pure $\mathrm{CuGeO}_{3}$ at the $\mathrm{Cu} K$-edge, and for $6 \% \mathrm{Ni}, 1.5 \% \mathrm{Mn}$ and $4 \% \mathrm{Zn}$ doped samples, at the impurity $K$-edges and (b) for pure $\mathrm{CuGeO}_{3}$ at the $\mathrm{Ge} K$-edge and for $2 \%$ Si-doped sample at the Si $K$-edge.

phase $\left(T>T_{S P}\right)$ but not that of Mg-doping because of the too low energy absorption of the $\mathrm{Mg}$ atom.

The next section is devoted to the experimental details and to a brief recall of the EXAFS analysis methodology. The EXAFS results are reported in section III for $\mathrm{Cu}$ site and Ge site doping. The structural distortions, as deduced from the EXAFS refined parameters, are analyzed in section IV. Their influence is discussed in section V, in relation with the magnetic properties, and a conclusion is given in Sec. VI.

\section{Experiment}

The EXAFS measurements at the $\mathrm{Cu}$ and $\mathrm{Ge}$ absorption $K$-edges, in transmission detection mode, and at the $\mathrm{Ni}$, $\mathrm{Mn}$ and $\mathrm{Zn}$ absorption $K$-edges, in fluorescence detection mode, were carried out at LURE (Orsay) on the D42 and D44 beamlines of the DCI storage ring. The experiments were performed at $78 \mathrm{~K}$, in the paramagnetic phase, above the spin-Peierls transition. The incident energy was varied by $2 \mathrm{eV}$ steps using a $\mathrm{Si}(111)$ single crystal monochromator. The EXAFS oscillations were obtained in transmis- sion detection mode by measuring the X-ray beam intensities with ionization chambers, before $\left(I_{0}\right)$ and after $\left(I_{1}\right)$ the sample, in an energy range close to the absorption $K$-edge of $\mathrm{Cu}\left(\lambda_{K}^{C u}=8979 \mathrm{eV}\right)$ and $\mathrm{Ge}\left(\lambda_{K}^{G e}=11103 \mathrm{eV}\right)$. The samples studied in the transmission detection mode were pellets of homogeneous thickness made of a mixture of about $15 \mathrm{mg}$ of single crystal ground into very fine powder and $35 \mathrm{mg}$ of cellulose. In the fluorescence detection mode, the powder obtained from ground doped crystals was spread on an adhesive tape. The EXAFS spectra were measured in the fluorescence detection mode with a multielements detector around the impurities $K$-edges in the $2 \%$ and the $6 \% \mathrm{Ni}$-doped samples (absorption $K$-edge $\left.\lambda_{K}^{N i}=8333 \mathrm{eV}\right), 1.5 \%$ Mn-doped sample $\left(\lambda_{K}^{M n}=6539\right.$ $\mathrm{eV})$, and the $4 \%$ Zn-doped sample $\left(\lambda_{K}^{Z n}=9659 \mathrm{eV}\right)$.

The Si $K$-edge EXAFS experiment was performed on the SA32 beamline of SUPERACO using a 2 InSb crystals monochromator. A thin slice of $2 \%$ Si-doped single crystal (absorption $K$-edge $\lambda_{K}^{S i}=1839 \mathrm{eV}$ ) was cleaved and stuck with $\mathrm{Ag}$ glue on an indium covered sample-holder. The absorption signal was recorded with a monoelement detector in the fluorescence detection mode.

The EXAFS oscillations were analyzed using the FEFF package [19]. The EXAFS oscillations, $\chi(k)$, were extracted from the experimentally measured absorption coefficient $\mu(E)$ using the AUTOBK program with $k$, the wavenumber of ejected photoelectron, equal to:

$$
k=\sqrt{2 m\left(E-E_{0}\right) / \hbar^{2}}
$$

where $E_{0}$ is the absorption energy for the $K$-edge and $m$ the electron mass.

For an absorbing central atom surrounded by $N$ identical atoms, when considering only single scattering paths, EXAFS oscillations created by the backscattering of the photoelectron from the neighboring atoms are given by:

$$
\begin{array}{r}
\chi(k)=-S_{0}^{2} \frac{N}{k r^{2}}|F(k, \pi)| e^{-2 k^{2} \sigma^{2}} e^{-2 r / \lambda(k)} \\
\sin [2 k r+2 \delta(k)+\Phi(k)]
\end{array}
$$

where $r$ is the mean pair distance, $\sigma^{2}$ is the associated Debye-Waller factor which takes into account the structural and dynamical disorders, and $S_{0}^{2}$ is the amplitude reduction factor accounting for many-body effects within the absorbing central atom. The other parameters in Eq. (2) are the phase $\Phi(k)$ and the modulus of the complex function $F(k, \pi)$, which both depend on the nature of the backscattering atom, the mean free path $\lambda(k)$ and the absorbing atom phase shift $\delta(k)$. They are derived from the theoretical ab-initio multiple scattering calculations by the FEFF program using crystallographic data. In order to describe a real environment, the surrounding atoms are gathered into atomic shells (atoms at approximately the same distance from the absorber), themselves subdivided into several subshells, for each kind of chemically and crystallographically equivalent neighbors.

Representative measured EXAFS oscillations $\chi(k)$ are shown in Fig. 3. Note that the spectra of the doped samples, recorded at the impurity $K$-edge, are more noisy than 


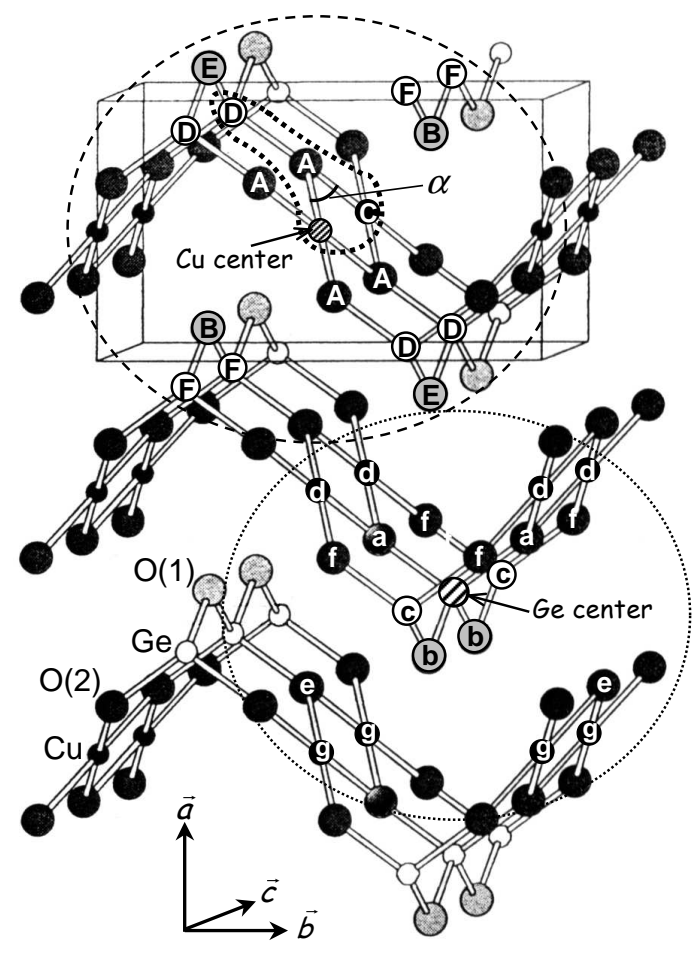

Fig. 4. Structure of $\mathrm{CuGeO}_{3}$. The $\mathrm{Cu}$ and Ge environments are materialized: starting from the dashed $\mathrm{Cu}$ and $\mathrm{Ge}$ absorbers, the atoms labelled "A","B", ... "F" (for $\mathrm{Cu}$ ) and "a", "b", ... "g" (for Ge) are their neighbors going from the closest to the furthest ones that were accessible in the EXAFS experiment.

those recorded at the $\mathrm{Cu}$ and $\mathrm{Ge} K$-edges because of the small amount of absorbers and of the fluorescence detection mode. In consequence, the EXAFS analysis could not be performed up to such large $k$ values at the impurity $K$-edges compared to the $\mathrm{Cu}$ and $\mathrm{Ge}$ ones. For the Si $K$ edge spectrum, an additional source of noise was the low fluorescence yield and the small photon flux at this low energy. The complex Fourier transforms (denoted FT) of the $k^{3}$ weighted $\chi(k)$ functions were then calculated using a Hanning window. The amplitude of this FT can be associated with a pseudo-radial atomic distribution around the absorber in the $r$-space. Lastly, the EXAFS oscillations of the atomic shells of interest have been isolated by a subsequent inverse Fourier-transform (denoted IFT) performed in a restricted appropriate $r$-range and leading to a Fourier filtered $k^{3} \chi(k)$ function. The FT and IFT were simulated with the FEFFIT program by summing the contributions given in Eq. (2) for all subshells obtained from the structural model. The multiple scattering paths, whose contribution was checked to be weak, were not included in the calculation. In the fit, the pair distances, the Debye-Waller factors and the energy shift $\Delta E_{0}$ were allowed to vary ${ }^{2}$. For the analysis of the EXAFS oscillations recorded at the

${ }^{2} \Delta E_{0}$ accounts for inaccuracies in the FEFF determination of the absorption threshold energy $E_{0}$. Different values of $\Delta E_{0}$ were refined for the $\mathrm{O}, \mathrm{Cu}$ and Ge neighbors. These extra shifts
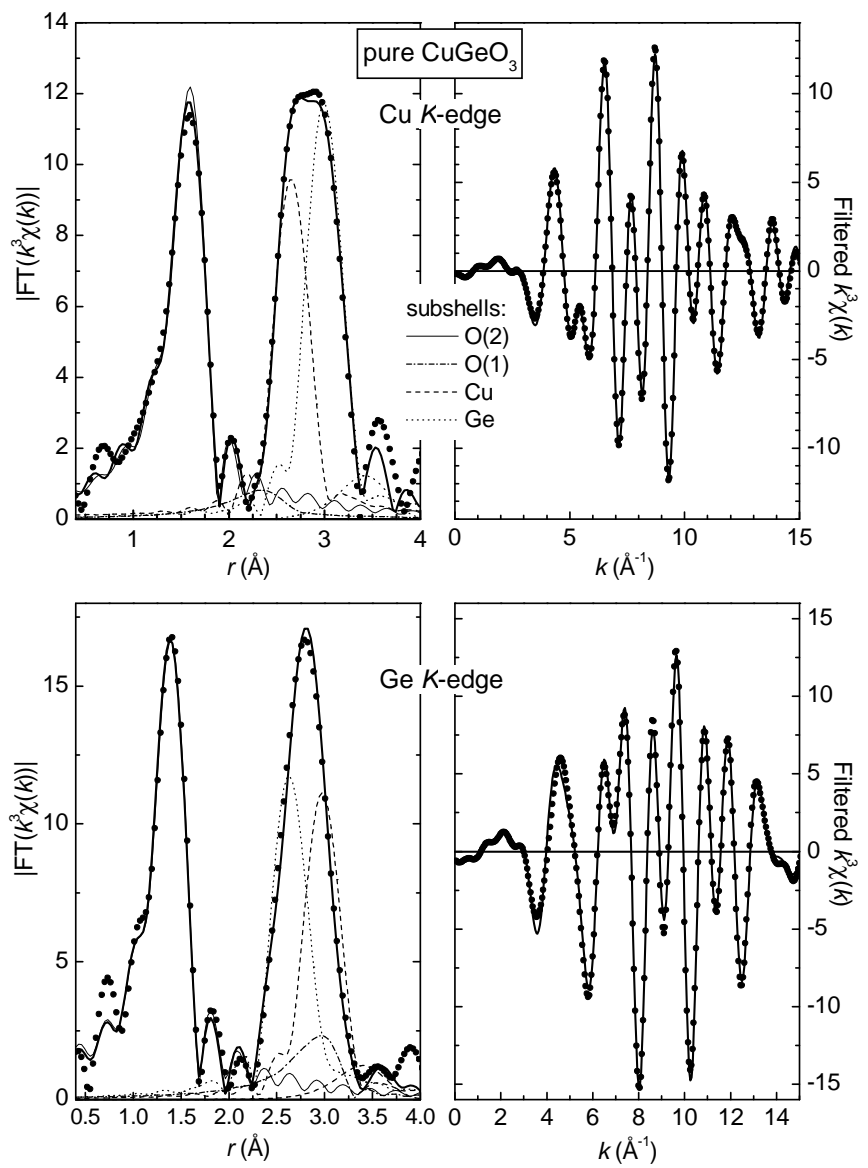

Fig. 5. EXAFS results for the pure $\mathrm{CuGeO}_{3}$ sample. Amplitude of the FT of $k^{3} \chi(k)$ (left) and Fourier filtered $k^{3} \chi(k)$ (right) at the $\mathrm{Cu}$ (top) and Ge (bottom) $K$-edges. The measured spectra are represented by full circles, the fit by a thick solid line. The contributions of each kind of neighbors are also shown on the FT plots (left).

impurity absorption edges, the FEFF files for each scattering path were recalculated with the impurity replacing $\mathrm{Cu}$ or $\mathrm{Ge}$ absorber within the same structure.

\section{Results}

\subsection{Pure sample}

The structure of $\mathrm{CuGeO}_{3}$ in the paramagnetic state was initially refined by Völlenkle et al. 20] and later by Braden et al. 17. 21] (space group Pbmm, $a=4.796 \AA, b=$ $8.466 \AA, c=2.940 \AA$ at $295 \mathrm{~K}$ ), from Rietvelt refinement of X-ray and neutron powder diffractograms ${ }^{3}$. The crystallographic data of Braden et al. [17] were used as input

are meant to correct errors introduced by FEFF in the calculation of the scattering phase shifts.

3 The structure was revised by M. Hidaka et al., J. Phys.: Condens. Matter 9, 809 (1997). They found a different space group $P 2_{1} 2_{1} 2_{1}$ and a larger unit cell $2 a \times b \times 4 c$ than Braden et 
of the FEFF program in our EXAFS analysis. These structural parameters yield, for the atomic distribution around $\mathrm{Cu}$ atoms, a first shell with the 4 square planar $\mathrm{O}(2)$ at $1.942 \AA$ and a second shell including the 2 apical $\mathrm{O}(1)$ at $2.78 \AA$, the 2 chain neighbors $\mathrm{Cu}$ at $2.95 \AA$ and $4 \mathrm{Ge}$ at $3.30 \AA$ (top of Fig. 4 ). The atomic distribution around Ge atoms is made of a first shell with the $4 \mathrm{O}$ of the tetrahedron $(2 \mathrm{O}(2)$ at $1.737 \AA$ and $2 \mathrm{O}(1)$ at $1.778 \AA)$ and a second shell including $2 \mathrm{Ge}$ at $2.946 \AA$ and $4 \mathrm{Cu}$ at $3.30 \AA$ (cf. bottom of Fig. 1). This shell description is summarized in Table I.

The EXAFS fitting procedure was tested on the pure $\mathrm{CuGeO}_{3}$ sample. The first two atomic shells of the radial distributions at both $\mathrm{Cu}$ and $\mathrm{Ge} K$-edges were simultaneously fitted. This implies that the distance and the Debye-Waller factor corresponding to each $\mathrm{Ge}-\mathrm{Cu}$ paths were hold identical for the two sets of data during the refinement. The $k$ range used for the FT and for the fitting procedure was $\left[3.5,15 \AA^{-1}\right]$ while the $r$ range used for the IFT was $[1,3.4 \AA]$ at both edges. The fits are in good agreement with the experimental spectra, as shown in Fig. 5, where the contribution of each kind of neighbors is represented. Note that, at the $\mathrm{Cu} K$-edge, the signal from the two apical oxygens $\mathrm{O}(1)$ is already very weak and the further $\mathrm{O}(1)$ neighbors were not considered because yielding a negligibly small contribution. However, the presence of the next 4 Ge atoms at about $3.786 \AA$ was found necessary to fit correctly the second shell. The resulting structural parameters obtained at both edges are listed in Table I and the comparison with their initial values shows the consistency of the fit. After this first EXAFS analysis, the same procedure was applied to the doped samples.

\subsection{Substitution on the $\mathrm{Cu}$ site}

The $\mathrm{Ni}$ and $\mathrm{Mn} K$-edges EXAFS oscillations were analyzed up to $k=12 \AA^{-1}$ (see Fig. 3). In the case of $\mathrm{Zn}$, an additional difficulty arose from the mixing of $\mathrm{Cu} K$-edge and $\mathrm{Zn} K$-edge EXAFS oscillations due to the closeness of the absorption energies of both elements: $8979 \mathrm{eV}$ for $\mathrm{Cu}$ and $9659 \mathrm{eV}$ for $\mathrm{Zn} .{ }^{4}$. Therefore, the $\mathrm{Zn} K$-edge spectrum, after correction from the contamination by the $\mathrm{Cu}$ $K$-edge oscillations, could be analyzed in a more limited $k$ range, up to $9 \AA^{-1}$ (cf. Fig. 3).

First, the structural results derived at the $\mathrm{Cu} K$-edge in the doped samples were found identical to those of the pure sample, even for doping levels as high as $6 \%(\mathrm{Ni})$. The distortions induced by the impurities were then deduced from the comparison of the structural parameters

al. [17, who nonetheless reconfirmed the initial structure one year later 21.

4 To correct the $\mathrm{Zn} K$-edge spectrum, the $\mathrm{Cu} K$-edge oscillations $\chi(E)$ of the pure sample, recorded up to $9998 \mathrm{eV}$, were converted into $\chi(k)$ using Eq.(11) with the absorption energy $E_{0}$ of $\mathrm{Zn}$. The $\mathrm{Cu}$ EXAFS contribution, whose amplitude at the $\mathrm{Zn}$ edge was obtained from the ratio of both absorption jumps recorded in a unique scan in the doped sample, was then subtracted from the Zn spectrum.
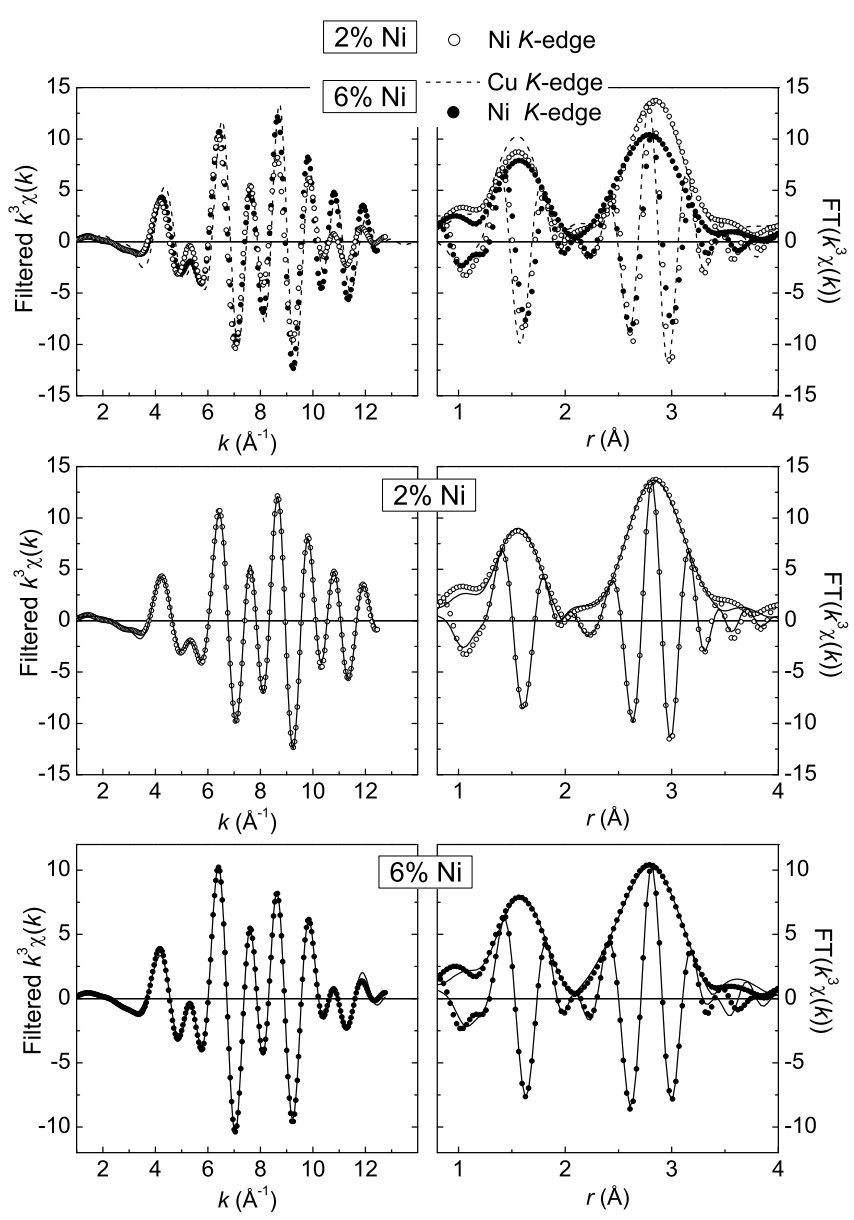

Fig. 6. EXAFS results for the $2 \%$ and $6 \% \mathrm{Ni}$-doped $\mathrm{CuGeO}_{3}$. Amplitude and imaginary part of the FT of $k^{3} \chi(k)$ (right) and Fourier filtered $k^{3} \chi(k)$ (left). Comparison of the measured spectra at the Ni $K$-edge for both doping levels and at the $\mathrm{Cu}$ $K$-edge for $6 \% \mathrm{Ni}$ sample (top). Comparison of the measured spectra (circles) and of the fits (solid lines) at the Ni K-edge for $2 \%$ doping (middle) and 6\% doping (bottom).

obtained at the impurity and at the $\mathrm{Cu} K$-edge in the same sample and using the same $k$ and $r$-ranges of analysis for the EXAFS oscillations. Note that we did not try to take into account impurity neighbors in our structural model (although there can be some for high doping levels) because it is most probably impossible to distinguish them from $\mathrm{Cu}$ neighbors in the EXAFS analysis.

The FT of $k^{3} \chi(k)$ and Fourier filtered $k^{3} \chi(k)$ are shown in Fig. 6 for the $2 \%$ and $6 \%$ Ni-doped compounds and in Fig. 7 for the $1.5 \% \mathrm{Mn}$ and $4 \% \mathrm{Zn}$-doped compounds. The spectrum recorded at the impurity $K$-edge in each compound is compared to its fit and to the reference spectrum at the $\mathrm{Cu} K$-edge. The structural parameters extracted from the fits are reported in Table II. The fits in $k$ and $r$ spaces are of rather good quality. For all doping values, the amplitude of the FT, for the first O shell, is smaller at the impurity $K$-edge than at the $\mathrm{Cu}$ one, which is related 
Table 1. Structural parameters of the atomic radial distribution up to 3.95 Åcentered on $\mathrm{Cu}$ and Ge atoms, from the crystallographic data of Braden et al. [17 and from the EXAFS simulations of the pure $\mathrm{CuGeO}_{3}$ sample at both $\mathrm{Cu}$ and $\mathrm{Ge} K$-edges. The atom labels are those of Fig. 1. Crystallographic data of Braden et al. [17] imply two slightly different Ge-O distances for the two pairs of oxygens within the tetrahedron. The parameters reported with no error bar were held fixed during the fit. The error bars only account for the statistical uncertainties and are therefore probably underestimated. The pair distances $r$ and Debye-Waller factors $\sigma^{2}$ are in $\AA$ and $\AA^{2}$ respectively. The quality of the EXAFS fit is given by the $R$-factor: $R f=0.002$ at the $\mathrm{Cu} K$-edge and $R f=0.008$ at the Ge $K$-edge. The refined $S_{0}^{2}$ values are 0.927 at the $\mathrm{Cu} K$-edge and 0.953 at the Ge $K$-edge.

\begin{tabular}{|c|c|c|c|c|c|c|c|c|c|}
\hline \multicolumn{2}{|c|}{$\overline{\mathrm{Cu} \text { center }}$} & Ref. [17] & \multicolumn{2}{|c|}{ EXAFS } & \multicolumn{2}{|c|}{ Ge center } & \begin{tabular}{l||l|} 
Ref. & 17
\end{tabular} & \multicolumn{2}{|c|}{ EXAFS } \\
\hline atom & label & $r$ & $r$ & $\sigma^{2}$ & atom & label & $r$ & $r$ & $\sigma^{2}$ \\
\hline $4 \mathrm{O}(2)$ & $\mathrm{A}$ & 1.9324 & $1.942(5)$ & $0.0032(4)$ & $2 \mathrm{O}(2)$ & $\mathrm{a}$ & 1.7322 & $1.737(3)$ & $0.0022(3)$ \\
\hline $2 \mathrm{O}(1)$ & B & 2.7547 & $2.77(4)$ & $0.010(6)$ & $2 \mathrm{O}(1)$ & $\mathrm{b}$ & 1.7729 & $1.778(3)$ & $0.0022(3)$ \\
\hline $2 \mathrm{Cu}$ & $\mathrm{C}$ & 2.9400 & $2.944(7)$ & $0.0027(4)$ & $2 \mathrm{Ge}$ & $\mathrm{c}$ & 2.9400 & $2.941(5)$ & $0.0022(3)$ \\
\hline $4 \mathrm{Ge}$ & $\mathrm{D}$ & 3.2872 & $3.294(7)$ & $0.0040(2)$ & $4 \mathrm{Cu}$ & d & 3.2872 & $3.294(7)$ & $0.0040(3)$ \\
\hline $2 \mathrm{O}(1)$ & $\mathrm{E}$ & 3.6975 & & & $2 \mathrm{O}(2)$ & $\mathrm{e}$ & 3.3015 & 3.301 & $0.006(4)$ \\
\hline & & & & & $4 \mathrm{O}(2)$ & $\mathrm{f}$ & 3.4123 & 3.412 & $0.006(4)$ \\
\hline $4 \mathrm{Ge}$ & $\mathrm{F}$ & 3.7717 & $3.74(3)$ & $0.014(2)$ & $4 \mathrm{Cu}$ & $\mathrm{g}$ & 3.7717 & $3.74(3)$ & $0.014(2)$ \\
\hline
\end{tabular}
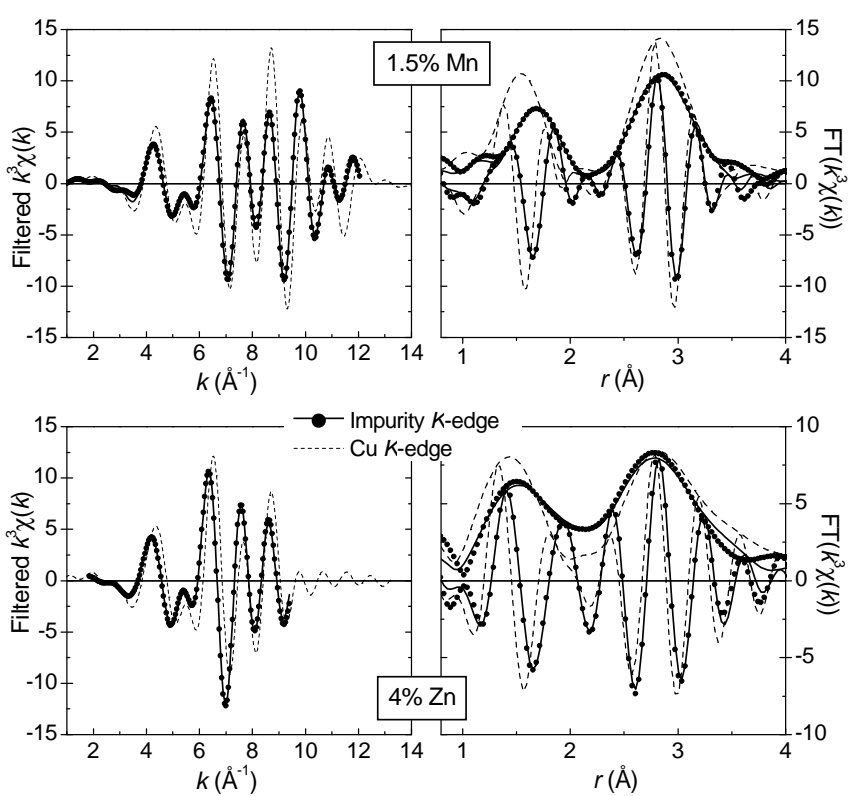

Fig. 7. EXAFS results for $1.5 \% \mathrm{Mn}$ (top) and $4 \% \mathrm{Zn}$ (bottom) doped $\mathrm{CuGeO}_{3}$ : amplitude and imaginary parts of the FT of $k^{3} \chi(k)$ (right) and Fourier filtered $k^{3} \chi(k)$ (left) of the measured data (full circles) and their fits (solid lines) at the impurity $K$-edge. The $\mathrm{Cu} K$-edge spectra measured on the same sample are also shown (dashed lines), for comparison.

to an increase of the Debye-Waller factor, in agreement with a doping-induced structural disorder. An increased Debye-Waller factor is also observed for the second shell in the case of the $6 \% \mathrm{Ni}$ and $1.5 \% \mathrm{Mn}$-doped compound. Note that the $4 \%$ Zn-doped compound shells are less welldefined because of the smaller $k$ range of integration. For all compounds, the first shell is shifted towards larger $r$ values. The distance from the absorber to the $\mathrm{O}(2)$ first neighbors increases more and more when replacing $\mathrm{Cu}$ by $\mathrm{Ni}(\Delta r / r=1.4 \%$ and $2.2 \%$ for 2 and $6 \%$ doping resp.),
$\operatorname{Zn}(\Delta r / r=4.5 \%)$ and $\operatorname{Mn}(\Delta r / r=7.3 \%)$. Interestingly, the structural distortion varies also for a same impurity as a function of its doping level, as shown by the increase of the Debye-Waller factor and first $\mathrm{Ni}-\mathrm{O}(2)$ distance from $2 \%$ to $6 \% \mathrm{Ni}$. For $\mathrm{Ni}$ and $\mathrm{Zn}$ impurities, there is an ambiguity concerning the position of the next nearest neighbors, the apical oxygens $\mathrm{O}(1)$. They are found either closer or further away from the impurity than from $\mathrm{Cu}$ (see parameters of the equally good EXAFS fits in Table II). At the Mn $K$-edge, there is no such ambiguity since the EXAFS spectra is 10 times better fitted when the $\mathrm{O}(1)$ atoms are closer from the impurity than further away, as compared to the $\mathrm{Cu}$ environment. Lastly, there is a clear variation of the distances to the next $\mathrm{Cu}$ and $\mathrm{Ge}$ neighbors with respect to the $\mathrm{Cu}$ environment $(1.6 \%$ and $1 \%$ increase, respectively) in the case of the $\mathrm{Mn}$ impurity only.

\subsection{Substitution on the Ge site}

The EXAFS oscillations, recorded at the Si $K$-edge in a $2 \%$ Si-doped sample, are limited to $8.7 \AA^{-1}$ and allow getting information only about the first $\mathrm{O}$ tetrahedral shell. Note that, due to the small range of fitting, the DebyeWaller factor could not be accurately refined and was fixed to $0.002 \AA^{2}$, i.e. to the value found in the pure sample at the Ge $K$-edge. This may be one reason for the small discrepancies ( $R$-factor value of 0.011 ) observed between the fit and the experimental data (Fig. 8). The results, obtained using $S_{0}^{2}=0.81$ in the fitting procedure, indicate a strong contraction of the $\mathrm{Si}-\mathrm{O}$ distance of about $8.4 \%$ with respect to the $\mathrm{Ge}-\mathrm{O}$ distance. In addition, a more symmetric environment is found around the Si than around the Ge atoms, that can be described by a small regular $\mathrm{O}$ tetrahedron with distance from center to corner of 1.61(2) $\AA$ (instead of 1.74 and $1.78 \AA$ for the two pairs of oxygen forming the distorted tetrahedron surrounding the Ge atoms). 
Table 2. Structural parameters obtained from the simulations of the EXAFS spectra at the impurities $K$-edges of the Cusite doped $\mathrm{CuGeO}_{3}$ samples: $R$-factors (quality of the fit), relative distance variations $\Delta r / r$ and Debye-Waller factors $\sigma^{2}$. The relative distance variations induced by impurities are calculated with respect to the results obtained for the Cu $K$-edge spectrum of the same sample analyzed using the same $k$ and $r$ ranges (see Table 1). For Ni and Zn impurities, two roughly equivalent solutions of the EXAFS fits are reported with a different distance from the impurity to the apical oxygens $\mathrm{O}(1)$, while only one solution is relevant for $\mathrm{Mn}$. The results given in bold characters correspond to the solutions validated by the analysis presented in Sec. 4.1. Note that the parameters presented with no error bar were held fixed during the fit and equal to the Cu $K$-edge refined value in the same sample. The $S_{0}^{2}$ factors at all the impurities $K$-edges were fixed to the value of 0.927 obtained at the $\mathrm{Cu} K$-edge.

\begin{tabular}{|c|c|c|c|c|c|c|c|c|}
\hline & & \multicolumn{2}{|c|}{$2 \% \mathrm{Ni}$} & \multicolumn{2}{|c|}{$6 \% \mathrm{Ni}$} & \multicolumn{2}{|c|}{$4 \% \mathrm{Zn}$} & $1.5 \% \mathrm{Mn}$ \\
\hline \multicolumn{2}{|l|}{$\overline{R f}$} & 0.0021 & 0.0023 & 0.0025 & 0.0058 & 0.0015 & 0.0030 & 0.0016 \\
\hline \multirow{5}{*}{$\frac{\Delta r}{r}(\%)$} & $\mathrm{O}(2)$ & $1.4(7)$ & $1.3(6)$ & $2.2(7)$ & $2.2(7)$ & $4.5(1.8)$ & $3.9(1.3)$ & $7.3(6)$ \\
\hline & $\mathrm{O}(1)$ & $-17(4)$ & $4(4)$ & $-15(2)$ & $4(4)$ & $-15(3)$ & $9(2)$ & $-13(1)$ \\
\hline & $\mathrm{Cu}$ & $0.6(6)$ & $0.7(7)$ & $-0.3(6)$ & $0.1(8)$ & $-0.9(8)$ & $-0.7(1.0)$ & $1.6(6)$ \\
\hline & $\mathrm{Ge}$ & $0.0(4)$ & $0.15(70)$ & $0.1(5)$ & $0.4(7)$ & $0.3(1.6)$ & $1.3(1.2)$ & $1.0(6)$ \\
\hline & $\mathrm{Ge}$ & 0.0 & 0.15 & 0.1 & 0.4 & 0.3 & 1.3 & 1.0 \\
\hline \multirow{5}{*}{$\sigma^{2}\left(\AA^{2}\right)$} & $\mathrm{O}(2)$ & $0.0038(4)$ & $0.0039(4)$ & $0.0042(4)$ & $0.0042(5)$ & $0.0035(11)$ & $0.0044(6)$ & $0.0045(3)$ \\
\hline & $\mathrm{O}(1)$ & $0.025(12)$ & $0.017(13)$ & $0.0119(4)$ & 0.013 & $0.017(13)$ & 0.01 & $0.0088(20)$ \\
\hline & $\mathrm{Cu}$ & $0.0034(8)$ & $0.0032(9)$ & $0.0038(6)$ & $0.0035(8)$ & 0.002 & 0.002 & $0.0047(10)$ \\
\hline & $\mathrm{Ge}$ & $0.0032(4)$ & $0.0034(5)$ & $0.0045(4)$ & $0.0049(6)$ & 0.0045 & 0.0045 & $0.0048(4)$ \\
\hline & $\mathrm{Ge}$ & $0.021(11)$ & $0.025(19)$ & $0.012(3)$ & $0.014(6)$ & 0.014 & 0.014 & $0.026(16)$ \\
\hline
\end{tabular}

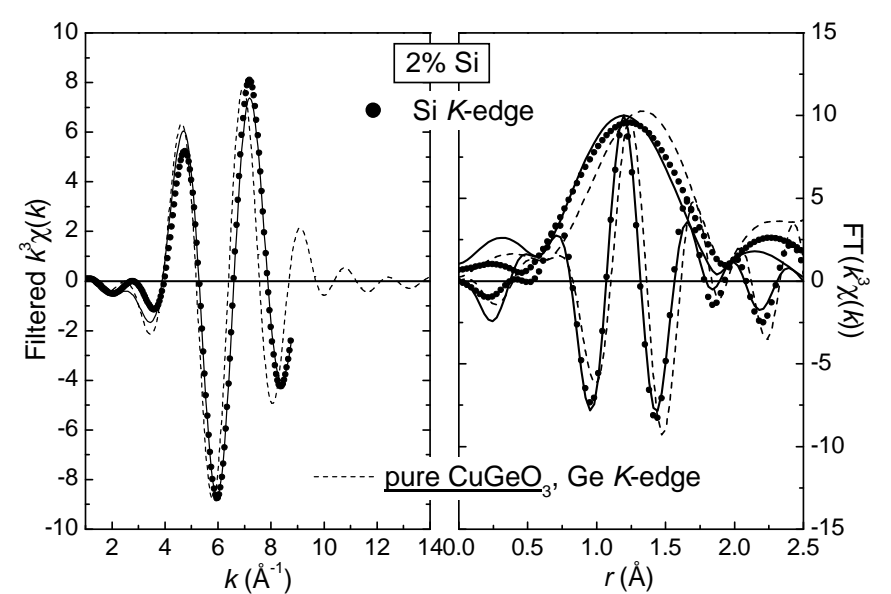

Fig. 8. EXAFS results for $2 \%$ Si-doped $\mathrm{CuGeO}_{3}$. Amplitude and imaginary part of the FT of $k^{3} \chi(k)$ (right) and Fourier filtered $k^{3} \chi(k)$ (left) at the Si $K$-edge of the doped sample (full circles) compared to the fit (solid lines) and to the results obtained at the Ge $K$-edge on the pure sample (dashed lines).

\section{Analysis}

\subsection{Comparison with ionic radii}

The ionic radius for each chemical species, which depends mainly on its oxydation and spin states, on its coordination number (the radius increases with the number of neighbors) and on the polyhedral description of its environment, can be roughly estimated as a function of these sole criteria and independently of the detailed structure [22]. It is therefore interesting to compare the results of the present experiment concerning the structural distor- tion of the first shell around the impurities with the pair distances evaluated from these ionic radii (tabulated in Table I of Ref. 22]). This qualitative analysis allows checking the validity of the EXAFS analysis and lifting some remaining ambiguities.

In practice, the $M-\mathrm{O}$ pair distance ( $M$ denoting the absorbing ion on the $\mathrm{Cu}$ or Ge site) is evaluated by summing the ionic radii of $M$ and of its $\mathrm{O}^{2-}$ first neighbors for comparison with the distances obtained from EXAFS. The results are summarized in Table III. The simplest case concerns the Ge site in which $\mathrm{Ge}^{4+}$ and $\mathrm{Si}^{4+}$ ions are surrounded by a slightly distorted and a regular tetrahedron, respectively. The calculations of the Ge-O and Si-O distances determined from EXAFS are in excellent agreement with the distances derived from the ionic radii [22].

The case of the $\mathrm{Cu}$ site is more complex. The $\mathrm{Cu}^{2+}$ is surrounded by an elongated octahedron made of a distorted basal square of $4 \mathrm{O}(2)$ and 2 apical $\mathrm{O}(1)$ at a much larger distance. The calculated $\mathrm{Cu}-\mathrm{O}$ distance from the ionic radii, in the hypothesis of square planar configuration, yields a very good agreement with the measured $\mathrm{Cu}-$ $\mathrm{O}(2)$ distance indicating that the orbital configuration of the $\mathrm{Cu}$ is mainly sensitive to the 4 closer $\mathrm{O}(2)$. According to the EXAFS results, there are two indistinguishable solutions for the geometry of the oxygen octahedra around $\mathrm{Ni}^{2+}$ and $\mathrm{Zn}^{2+}$ impurities on the $\mathrm{Cu}$ site. The apical $\mathrm{O}(1)$ would get closer to the impurity or further away as compared to the $\mathrm{Cu}$ environment, corresponding to an environment closer to a regular octahedron or closer to a square planar environment, respectively. Note however that a square planar configuration exists only for $\mathrm{Cu}$ and $\mathrm{Ni}$. Concerning the $\mathrm{Ni}$, the only way to reproduce the observed increase of the $\mathrm{Ni}-\mathrm{O}(2)$ distance, as compared to the $\mathrm{Cu}$ one, is an orbital configuration compatible with an $\mathrm{O}$ octahedron around the $\mathrm{Ni}$ and excludes a square 
Table 3. Distances to the first O neighbors, in $\AA$, calculated from the ionic radii ( ${ }^{a}$, from Ref. [22]) in octahedral and in square planar environments (when possible) for the $\mathrm{Cu}$ site, and in tetrahedral environment for the Ge site. The distances in bold are compatible with those derived from EXAFS and reported below $\left(^{b}\right)$ with the corresponding elongation ratio of the octahedron. The $2 \%$ and $6 \%$ Ni-doping results are reported. The last line of both tables gives the superexchange $\alpha$ angle in degrees, estimated from these EXAFS results (see Sec. 4.2).

\begin{tabular}{l|ccccc|cc}
\hline \hline & $\mathrm{Cu}$ & $\mathrm{Ni}$ & $\mathrm{Mg}$ & $\mathrm{Zn}$ & $\mathrm{Mn}$ & $\mathrm{Ge}$ & $\mathrm{Si}$ \\
\hline planar square $^{a}$ & $\mathbf{1 . 9 2}$ & 1.84 & & & & & \\
octahedron $^{a}$ & 2.08 & $\mathbf{2 . 0 4}$ & $\mathbf{2 . 0 7}$ & $\mathbf{2 . 0 9}$ & $\mathbf{2 . 1 8}$ & & \\
tetrahedron $^{a}$ & & & & & & $\mathbf{1 . 7 4}$ & $\mathbf{1 . 6 1}$ \\
\hline $\mathrm{O}(2)^{b}$ & 1.942 & $1.96 / 1.98$ & & 2.03 & 2.08 & 1.737 & 1.61 \\
$\mathrm{O}(1)^{b}$ & 2.78 & $2.26 / 2.33$ & & 2.36 & 2.38 & 1.778 & 1.61 \\
$\frac{r(M / C u-O(1))}{r(M / C u-O(2))}$ & 1.43 & $1.15 / 1.18$ & & 1.16 & 1.14 & & \\
\hline$\alpha$ angle & $98.9(1)$ & $97.9(3)$ & $95.8(3)$ & $95.85(15)$ & $98.9(1)$ & $94(1)$ \\
\hline \hline
\end{tabular}

planar description. This conclusion also holds for $\mathrm{Zn}$, for which only an octahedral environment is expected to exist. For $\mathrm{Mn}^{2+}$, the EXAFS analysis was unambiguous. A $\mathrm{Mn}-\mathrm{O}$ distance close to the one derived from the EXAFS analysis is indeed found when the ionic radius of $\mathrm{Mn}^{2+}$ is evaluated assuming an octahedral environment and a high spin state (i.e. $\mathrm{S}=5 / 2$ ), as inferred from the fine structure splitting of the ESR spectra at low temperature [8]. Note that the $M-\mathrm{O}$ distances for $\mathrm{Ni}, \mathrm{Zn}$ and $\mathrm{Mn}$, are slightly smaller than the one derived from the ionic radii because the octahedron is distorted. Some characteristic features of elongated octahedral environments are also observed in the X-ray Absorption Near Edge Structure spectra measured at the $\mathrm{Cu}$ K-edge in the pure compound and at the $\mathrm{Cu}$-site impurities $\mathrm{K}$-edge in the doped compounds, in agreement with the present analysis. These considerations allow to validate the EXAFS solutions for the $M$ $\mathrm{O}$ distances and to reproduce qualitatively their increase when substituting $\mathrm{Cu}$ by $\mathrm{Ni}, \mathrm{Zn}$ and $\mathrm{Mn}$, in this order. For the non-magnetic Mg impurity, extensively studied but for which no EXAFS measurements could be done, the following prediction can be made based on the same ionic radii analysis: the distortions around $\mathrm{Mg}$ are expected to be intermediate between those induced by $\mathrm{Ni}$ and $\mathrm{Zn}$. Note that the distortion reduction of the oxygen octahedron, when replacing $\mathrm{Cu}$ by the other impurities, could be expected since the axial elongation in the $\mathrm{Cu}^{2+}$ case is related to a strong Jahn-Teller effect, usual for this ion. Its substitution with non-magnetic or non Jahn-Teller 3d ions, like $\mathrm{Ni}^{2+}$ or high spin $\mathrm{Mn}^{2+}$, should reduce this effect. The remaining octahedral distortion is then only induced by the structural environment.

To summarize, i- the $\mathrm{Cu}$ environment is closer to a square planar one $\left(r_{C u-O(1)} / r_{C u-O(2)} \simeq 1.4\right)$ while substituting this site yields an environment closer to a regular octahedron $\left(r_{M-O(1)} / r_{M-O(2)} \simeq 1.14-1.18\right.$ for $M=$ $\mathrm{Ni}, \mathrm{Zn}, \mathrm{Mn}$ ) with the first distance increasing with respect to $\mathrm{Cu}$ in the following order: $M=\mathrm{Ni}, \mathrm{Mg}, \mathrm{Zn}, \mathrm{Mn}$, ii- Si-doping on the Ge site induces a contraction of its surrounding oxygen tetrahedron, as strong as the pushing away of the $\mathrm{O}(2)$ first neighbors around the Mn impurity.

\subsection{Superexchange angle calculation}

The distance variations in the impurities environment determined from the present experiment can now be used to calculate a structural parameter relevant for the magnetic properties: the superexchange angle $\alpha$, connecting 2 adjacent $\mathrm{Cu}$ sites through the $\mathrm{O}(2)$ atom. In the case of doping on the $\mathrm{Cu}$ site, this angle $\alpha=M-\mathrm{O}(2)-\mathrm{Cu}$ will be modified within one chain on both sides of the impurity $M$, while in the case of doping on the Ge site, this angle $\alpha=\mathrm{Cu}-\mathrm{O}(2)-\mathrm{Cu}$ involves the $\mathrm{O}(2)$ connected to the $\mathrm{Si}$ impurity and will thus be altered on two adjacent chains along the $b$ direction (see Fig. 14).

Concerning the $\mathrm{Cu}$-site impurities $M=\mathrm{Zn}, \mathrm{Ni}$ and $\mathrm{Mn}$, two hypothesis for the direction of displacement of their nearest neighbors can be made in order to estimate $\alpha$ from the measured $r_{M-O(2)}$ and $r_{M-C u}$ distances. Note that the distance $r_{M-C u}$ is altered only in the case of $\mathrm{Mn}$ doping. In the first hypothesis, the $\mathrm{O}(2)$ are pushed away from the impurity in the same direction than the initial $\mathrm{Cu}-\mathrm{O}(2)$ one (cf. Fig. 9(a), arrow (1)). This also produces a slight modification of the distance between the $\mathrm{O}(2)$ and the first $\mathrm{Cu}$ neighbor of the impurity. In the second hypothesis, this $\mathrm{Cu}-\mathrm{O}(2)$ distance is held identical to that in the pure sample, provided a small modification of the $M-\mathrm{O}(2)$ direction is made (cf. Fig. 9(a), arrow (2)). This assumption is suggested by the rigidity of the $\mathrm{Cu}-\mathrm{O}(2)$ bond whose length is found unchanged in the otherwise largely distorted $\mathrm{CuSiO}_{3}$ compound 23, 24. The calculated $\alpha$ values with both hypothesis are $98-97.7^{\circ}$ for $2 \%$ $\mathrm{Ni}, 97.6-97.2^{\circ}$ for $6 \% \mathrm{Ni}, 96.1-95.5^{\circ}$ for $4 \% \mathrm{Zn}$, and $95.7-$ $96^{\circ}$ for $1.5 \% \mathrm{Mn}$ respectively, yielding the average values listed in Table III. The angle decreases for all impurities on the $\mathrm{Cu}$ site, with respect to their value in the pure compound $\left(99^{\circ}\right)$, but this effect is smaller in Ni-doped compounds than in the $\mathrm{Zn}$ and Mn-doped compounds.

In the case of substitution by $\mathrm{Si}$ on the Ge site, a complication arises from the fact that the whole geometry is not planar i.e. the $\beta$ angle between the $\mathrm{Cu}-\mathrm{O}(2)$ ribbons and the $\mathrm{Ge}-\mathrm{O}(2)$ segment is equal to $159.5^{\circ}$ (cf. Fig. $9(\mathrm{~b})$ ). A simple assumption for the Si-induced distortion is a contraction of the $\mathrm{O}$ tetrahedron around the impurity without change of bond direction with respect to the Ge environ- 
(a)

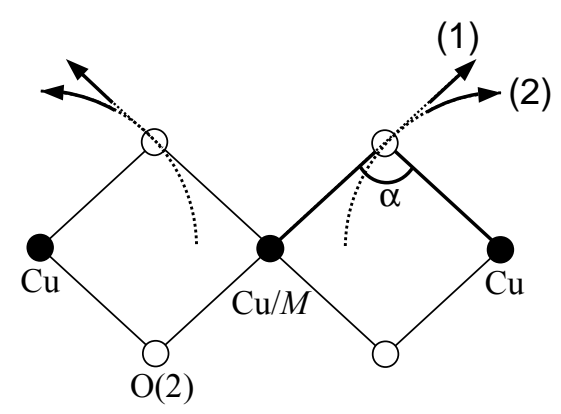

(b)

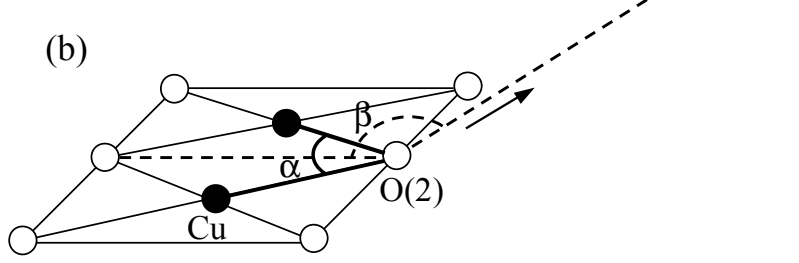

Fig. 9. Schematic view of the local deformation, due to $M / \mathrm{Cu}$ (a) and $\mathrm{Si} / \mathrm{Ge}$ (b) substitution, assumed for the calculation of the local $\mathrm{Cu}($ or $M)-\mathrm{O}(2)-\mathrm{Cu}$ angle $\alpha$ (see text for the different scenarii).

ment. In this case, the $\mathrm{O}(2)$ atom is pulled towards the Si atom by $0.13 \AA$ (arrow in Fig. $9(\mathrm{~b})$ ). This leads to an increase of the $\beta$ angle $\left(161.7^{\circ}\right)$ and a decrease of the $\alpha$ angle down to $94.1^{\circ}$. Note that a different variation of $\beta$ towards larger (resp. smaller) values would increase (resp. decrease) the value of $\alpha$ (estimated variation within \pm $1^{\circ}$ ). A possible contraction of the $\mathrm{Cu}-\mathrm{Cu}$ distance was not taken into account in this simple estimation of $\alpha$, while it is suggested from the study of Si-doped polycrystals by X-ray diffraction (variation of the $c$ parameter) [25]. This would decrease even more the $\mathrm{Cu}-\mathrm{O}(2)-\mathrm{Cu}$ angle. In conclusion, the reduction of the $\alpha$ angle should be even stronger for Si than for $\mathrm{Zn}$ and Mn-doping.

\section{Discussion}

\subsection{State of the art}

The next step is to relate the EXAFS results, and especially the above determination of the superexchange angle, to the magnetic properties of doped $\mathrm{CuGeO}_{3}$. Let us recall that there is a universal decrease of $T_{S P}$ versus the doping concentration with $y \approx 3 x$ in $\left(\mathrm{Cu}_{1-y} M_{y}\right)\left(\mathrm{Ge}_{1-x} \mathrm{Si}_{x}\right) \mathrm{O}_{3}$, whereas the lower temperature onset of an AF LRO occurs at different $T_{N}$ values depending on the impurity [5]. Two observations have stimulated a lot of theoretical work [26, 27, 28, 29, 30, 31]: the coexistence of both AF and SP LRO for small $\mathrm{Si}, \mathrm{Zn}, \mathrm{Ni}$ and $\mathrm{Mn}$ doping, as shown by neutron scattering studies [32, 33, 4, 34], and the apparent absence of critical concentration of impurities for the onset of this AF LRO 35. From the different models that have been proposed, a generally admitted description of the magnetic excitation of these dimerized chains emerges in terms of topological defects connecting regions of opposite dimerization parity. These defects, that can be introduced through doping, are then constituted by a soliton, pinned to the $\mathrm{Cu}$ substituent, followed on the same chain by an antisoliton which restores the interchain phasing. Although mobile in the isolated chains, the antisoliton remains confined close to the impurity site when interchain couplings are accounted for in order to minimize the size of the defective segment which presents an outof-phase dimerization with respect to the 3D pattern 36 . 28, 30. 31. Such a description of the solitonic defects, in terms of soliton-antisoliton pairs carrying an uncompensated spin $1 / 2$, leads to large AF fluctuations coexisting with underlying reduced dimerization. In pure samples, the spin-Peierls phase is established when the critical size and phase coherence of the dimerized domains is reached, allowing the system to collapse into a long-range ordered state. This average size of coherent dimerized domains is limited by thermally activated and/or doping-induced solitonic defects. This qualitatively explains the decrease of $\mathrm{T}_{S P}$ with increasing impurity concentration. On the other hand, in the presence of inter-chain coupling, the onset of an AF LRO occurs when the AF correlated regions associated with these doping-induced soliton-antisoliton pairs overlap.

The magnetic behavior under doping is thus qualitatively explained for non-magnetic impurities within the $\mathrm{Cu}$ chains. They simply cut one dimer, thus releasing one spin $1 / 2$ solitonic defect confined close to the impurity. However, this is less obvious in the case of magnetic impurities on the $\mathrm{Cu}$ site, which may still be magnetically coupled to the neighbor $\mathrm{Cu}$ spins, and even less for $\mathrm{Si}$ impurities that are introduced outside the $\mathrm{Cu}$ chains. The nature of the released soliton-antisoliton pair through doping is a key feature in the understanding of the establishment of the AF LRO. Its nature will crucially depend on the strength of the local magnetic interaction. It is therefore necessary to relate the structural parameters determined in the vicinity of the impurity in the previous sections with the value of the $\mathrm{Cu}-M$ exchange interaction. The variation of the in-chain nearest neighbor constant, $J_{1}$, with the $\mathrm{Cu}-\mathrm{O}(2)-\mathrm{Cu}$ superexchange angle $\alpha$ was inferred from the pressure dependence of the susceptibility in $\mathrm{CuGeO}_{3}$ and found to be equal to: $\delta J_{1} /\left(J_{1} \delta \alpha\right) \approx 5.8 \%$ [37, 38]. Various calculations based on the analysis of microscopic magnetoelastic coupling 39] and microscopic structural models 117 yielded different estimations: $\delta J_{1} /\left(J_{1} \delta \alpha\right) \approx 10 \%$ and $22 \%$, respectively. A value of $\delta J_{1} /\left(J_{1} \delta \alpha\right)$ ranging between 10 and $20 \%$ was also found in microscopic calculations of the exchange constants carried out using different approaches: perturbation theory, exact diagonalization of $\mathrm{Cu}_{2} \mathrm{O}_{2}$ clusters, and band calculations [38]. The general result of these studies is that $\alpha$ reaches its critical value, corresponding to the cancellation of $J_{1}$, i.e. to the transition from $\mathrm{AF}$ to $\mathrm{FM}$ coupling, between 90 and $95^{\circ}$. Other structural parameters can also affect $J_{1}$ like the angle $\beta$ between the $\mathrm{Cu}-\mathrm{O}(2)$ ribbons and the $\mathrm{Ge}-\mathrm{O}(2)$ segment, or the pair distances involved in the exchange path, and 
more generally, all the parameters that may influence the strength of the orbital hybridization.

\subsection{Antiferromagnetism vs distortions}

\subsubsection{In-chain magnetic impurities}

Concerning the magnetic impurities on the $\mathrm{Cu}$ site, let us recall that they drive the system towards an AF LRO at a temperature $T_{N}$ different from that induced by nonmagnetic $\mathrm{Cu}$-site impurities, slightly lower for $\mathrm{Ni}$ and larger for $\mathrm{Mn}$. From our estimation of the local $\alpha$ angle (Table III), the magnetic local coupling $J_{1}^{l o c}$ between $\mathrm{Ni}^{2+}$ (spin 1) and its $\mathrm{Cu}^{2+}$ neighbors in Ni-doped $\mathrm{CuGeO}_{3}$ is expected to be still AF but smaller than the coupling between $\mathrm{Cu}$ neighbors $\left(J_{1}=120-180 K\right)$. Both an analysis of the thermal dependence of the magnetic susceptibility [5] and a resonance model for ESR results [13 have indeed evidenced the formation of $\mathrm{Ni}-\mathrm{Cu}$ pairs with an $\mathrm{AF}$ coupling $J_{1}^{l o c}$ weaker than $J_{1}$ (most probable value of $J_{1}^{\text {loc }}$ estimated to be around $75 \mathrm{~K}[13$ ). The spin 1 of the $\mathrm{Ni}$ impurity and the released spin 1/2 of the $\mathrm{Cu}$ therefore form an effective spin $1 / 2$ solitonic defect at low temperature. In the case of Mn-doping, the $\alpha$ value for the $\mathrm{Mn}-\mathrm{O}(2)$ $\mathrm{Cu}$ path (Table III) suggests that $J_{1}^{\text {loc }}$, although still AF, becomes very weak, which could be reinforced by the frustrating $J_{2}$ next nearest neighbor interaction. Here again, some ESR measurements [8] support this result with the observation of an hyperfine spectrum of the ${ }^{55} \mathrm{Mn}$ (nuclear spin $\mathrm{I}=5 / 2$ ) with fully developed 6 lines for very low Mn concentration $(0.1 \%)$. An upper value of $J_{1}^{l o c} \simeq 4 \mathrm{mK}$ can be estimated from the splitting of the hyperfine lines. The detection of quasi free $5 / 2$ spins between $T_{S P}$ and $T_{N}$ thus demonstrates that the effective coupling between $\mathrm{Mn}^{2+}$ and $\mathrm{Cu}^{2+}$ is close to zero. Hence, the onset of the AF LRO is mainly due to a collective behaviour of the spins $1 / 2$ solitonic defects, decoupled from the free $5 / 2$ spins of the Mn impurities. It is indeed excluded for the direct $\mathrm{Mn}$ exchange to be responsible for the transition to AF LRO, due to the very large distances between the Mn atoms.

Therefore, the AF LRO transition observed for all the in-chain impurities presently studied seems to be mainly due to the cooperative behavior of soliton-antisoliton pairs (one released per impurity) carrying a spin $1 / 2$, whatever the magnetic state of the impurity. Now, another parameter can be considered in order to explain the differences between the various $T_{N}(y)$ transition lines. The present study has revealed differences in the amplitude of the distortion around each kind of impurities related to their ionic radii. For instance, the first neighbor distances to the impurity increases with respect to the $\mathrm{Cu}$ environment following the sequence $\mathrm{Ni} / \mathrm{Mg} / \mathrm{Zn} / \mathrm{Mn}$ (section 4.1). The strongest local distortion, in the case of Mn, clearly affects further neighbors (more than two $\mathrm{Cu}-\mathrm{Cu}$ distances). It is interesting to note that the $T_{N}$ values somehow vary in the same way. The distortions induced by the impurities along the $\mathrm{Cu}$ chains can be very large (Table II) compared to the atomic displacements produced by the dimerization itself :
$0.2 \%$ relative variation of the first $\mathrm{Cu}-\mathrm{Cu}$ distance. In consequence, they will destroy locally the in-chain dimerization and will render the energetic criterion for inter-chain phase coherence ineffective. This could in turn affect the spatial distribution of the soliton-antisoliton pairs along the chain axis, i.e. their overall size or/and the mobility of the antisoliton. It would become easier for the antisoliton to accommodate its distance from the impurity and the global size of the soliton-antisoliton pair could be enhanced. As a result, this additional degree of freedom may favor the overlap of the AF correlated regions and thus increase the $T_{N}$. This mechanism provides an explanation for the reported higher $T_{N}$ value for $\mathrm{Mn}$ with respect to $\mathrm{Zn}$ due to the larger structural distortion of its environment. On the contrary, for $\mathrm{Ni}$, the distortions are the smallest implying the lowest $T_{N}$, as observed. Furthermore, the AF coupling between the spin $1 \mathrm{Ni}$ and the spin $1 / 2 \mathrm{Cu}$ may pin the global spin $1 / 2$ soliton-antisoliton pair closer to the impurity. As a result, the overlap between the AF correlations is reduced and the $T_{N}$ is lowered. Lastly, close enough to $\mathrm{T}_{N}$, one can not rule out an indirect polarisation of the AF correlated regions by the $\mathrm{Mn}^{2+}$ spin (via the inter-chain interactions for instance), which could accelerate the transition towards the AF LRO.

\subsubsection{Out-of-chain Si impurity}

An unsolved issue remains concerning the strong influence of the out-of-chain Si-doping on the magnetic properties of $\mathrm{CuGeO}_{3}$. The present study has allowed us to determine the large local decrease of the superexchange angle $\alpha$ due to Ge substitution by the much smaller Si ion. $\alpha$ was found to be smaller than the one calculated in the case of Mndoping for which an almost vanishing $J_{1}^{\text {loc }}$ was inferred (Table III). This strongly suggests that $J_{1}^{l o c}$, coupling the spins of each $\mathrm{Cu}$ pair on both chains facing the Si impurity, is indeed ferromagnetic. This is in agreement with theoretical predictions of a weakly ferromagnetic value of $J_{1}^{l o c}$, which was calculated using realistic structural parameters: $\alpha=95^{\circ}$ and $\mathrm{Si}-\mathrm{O}$ bond length smaller by $0.13 \AA$ than the Ge-O one 18,38. The authors have invoked additional parameters enhancing this trend towards ferromagnetism: the further weakening of the hybridization of the $2 p_{y} \mathrm{O}(2)$ orbitals with $\mathrm{Si}$, with respect to $\mathrm{Ge}$, and the influence of frustrating $J_{2}$ next nearest interaction. This result is also comforted by the structure and magnetic properties of an isostructural $\mathrm{CuSiO}_{3}$ single crystal 23, 24. The $\alpha$ angle is equal to $94-95^{\circ}$ in this compound [23,40], i.e. close to the one determined locally around $\mathrm{Si}$ impurities in doped $\mathrm{CuGeO}_{3}$ (cf. section IV.B). The analysis, from magnetometry measurements and a neutron powder diffraction experiment, of the peculiar AF LRO arising around $8 \mathrm{~K}$ in $\mathrm{CuSiO}_{3}$, suggests a weak ferromagnetic nearest neighbor exchange interaction $J_{1}$ and a stronger antiferromagnetic next nearest neighbor interaction $J_{2}$, similar to the $\mathrm{CuGeO}_{3}$ one 23, 40].

Important consequences are expected from a ferromagnetic $J_{1}^{l o c}$ on the nature of the solitonic defects produced by the Si impurity and thus on the associated magnetic 
properties. The dimerization pattern imposes that the $\mathrm{Si}$ impurity faces one strong bond corresponding to a dimer and one weak bond between two dimers. One can reasonably assume that this local variation of $J_{1}$ will alter only the strong bond, freeing two spins $1 / 2$, and will have no effect on the other chain (even locally reinforcing its dimerization). In this case, the two released spins $1 / 2$ of the dimer, ferromagnetically coupled, may combine into an effective free spin 1.

The Curie constant, extracted from the temperature dependence of the susceptibility between $T_{S P}$ and $T_{N}$, in a series of $\mathrm{CuGe}_{1-x} \mathrm{Si}_{x} \mathrm{O}_{3}$ samples [7], was previously analyzed according to Eq. 3 in a simple model where local spins $S=1 / 2$ are associated with the solitonic defects.

$$
\chi_{\text {para }}(T)=K_{\text {para }} \frac{\left(g \mu_{B}\right)^{2}}{3 k_{B}(T-\theta)} S(S+1)
$$

with $g=2.05$, the Landé factor, $\mu_{B}$ the Bohr magneton, $k_{B}$ the Boltzman constant, $\theta$ the Curie-Weiss temperature, and $K_{\text {para }}=N x$ with $N$ the number of released effective spins $S$ per impurity. Once the contribution of spins $1 / 2$ due to intrinsic impurities has been subtracted, the number $N$ of spins $1 / 2$ per $\mathrm{Si}$ is found close to 3 , a puzzling value ${ }^{5}$. Assuming now that the solitonic defects are ferromagnetic pairs of spin $1 / 2$, the susceptibility of one pair is given by :

$$
\chi_{\text {pair }}(T)=\frac{\left(g \mu_{B}\right)^{2}}{k_{B} T} \frac{2}{3+\exp \left(-J_{1}^{l o c} / k_{B} T\right)}
$$

At low temperature, the expression of $\chi_{\text {para }}(T)$ then reduces to Eq. 3 for $N$ such magnetic entities with an effective spin $S=1$. This analysis yields $N \sim 1.2$, which is close enough to the expected value of one ferromagnetic pair of spins $1 / 2$ per Si impurity in this new description. This can be further argued from the analysis of the magnetization measured in a $0.3 \%$ Si-doped $\mathrm{CuGeO}_{3}$ sample (see Fig. 2 of Ref. 41). The $M(H)$ curve reaches a first saturation plateau $\vec{M}_{\text {sat }}=N x g \mu_{B} S=0.0077 \mu_{B}$ in the SP phase before entering the incommensurate phase. The analysis of the whole $M(H)$ curve with Brillouin functions, after correction for the intrinsic impurities contribution, yields $N \sim 1.1$ spins 1 or $N \sim 2.2$ spins $1 / 2$. The first result is in better agreement with the expected number of effective spins 1 and with the previous estimation of $N$, deduced from susceptibility analysis. The revised analysis of the magnetic measurements in Si-doped compounds is therefore in favor of the release of one solitonic defect per $\mathrm{Si}$ with an effective spin 1 , on one of both adjacent chains.

A consequence of the effective spin 1 of the solitonic defect produced by $\mathrm{Si}$ will be to renormalize $T_{N} \propto S(S+1)$ by a factor of 2.67 as compared to the case of spin $1 / 2$ solitonic defects released by the same concentration of $\mathrm{Cu}-$ site impurities. However, this comparison only holds if the spatial extension of the solitonic defect is otherwise identical, thus producing the same overlap between AF correlated regions. The overall size of the soliton-antisoliton

\footnotetext{
${ }^{5}$ Note that in Ref. [7], the values of $K_{\text {para }}$ were not corrected for the $0.11 \%$ intrinsic spin $1 / 2$ impurities found in pure $\mathrm{CuGeO}_{3}$ and expected to be also present in doped samples.
}

pair along the $c$-axis was indeed estimated to be the same for $\mathrm{Si}$ and $\mathrm{Zn}$ from X-ray measurements 42. This supported the origin of the factor $\sim 3$ between the $T_{N}$ of $\mathrm{Si}$ and of non-magnetic $\mathrm{Cu}$-site impurities from the different types of the effective spin associated to the solitonic defects, at least for low doping levels (see Fig. 2). The reason why this scaling does not hold at higher $x$ may be due to a different mechanism involved in the onset of AF LRO. The distances between impurities are becoming shorter than the 1D magnetic correlations, and moreover, a first-order transition is observed in the case of $\mathrm{Cu}$-site impurities between a dimerized and a uniform AF LRO, whereas the coherence length of the spin-Peierls ordering decreases progressively in the Si doping case 43.].

\subsection{Spin-Peierls transition vs distortions}

Contrary to the sensitivity of the AF LRO to the nature of the impurity, discussed in the previous section on the basis of the EXAFS results, the decrease of the spin-Peierls temperature appears to be more universal. On the $\mathrm{Cu}$ site, the decrease of $T_{S P}$ has exactly the same concentration dependence for all studied $\mathrm{Cu}$ substituents, whatever the spin impurity and the amplitude of the in-chain local distortions around impurities (see Fig. 2). For Si-doping on the Ge site, $T_{S P}$ decreases $\sim 3$ times faster with the concentration, although the size of the solitonic defect induced by $\mathrm{Si}$ in the chain direction is similar to the one induced by $\mathrm{Zn}$ impurities 42 .

Moreover, the average correlation length of the dimerized regions along $c$ does not seem to be so relevant for the appearance of the SP LRO, as shown by the measurements of fluctuations in pure and doped $\mathrm{CuGeO}_{3}$ for different impurities above the SP transition 44,,42]. First, the regime of $1 \mathrm{D}$ pretransitional fluctuations along the chain direction in pure $\mathrm{CuGeO}_{3}$ was shown to start well above $T_{S P}$, while these fluctuations become $2 \mathrm{D}$ and then $3 \mathrm{D}$ much closer to $T_{S P}$. Secondly, the average size of the dimerized regions along $c$ in the pretransitional regime for $\mathrm{Si}$, is comparable to that of $\mathrm{Zn}$ despite the factor $\sim 3$ in their $T_{S P}$. Last, the extension of the $1 \mathrm{D}$ pretransional fluctuations along $c$ is much smaller for $\mathrm{Ni}$ than for $\mathrm{Zn}$ or $\mathrm{Mg}$ 42, whereas their decrease of $T_{S P}$ has exactly the same doping level dependence.

Therefore, all these experimental results point out that the relevant parameter for the decrease of $T_{S P}$ through doping is not the way the dimerization is affected along the chain direction, but perpendicular to it through the stabilization of the out-of-phase 3D dimerization pattern. This is supported by a major difference observed concerning the spatial extension of the perturbation of the dimerized regions when doping inside and outside of the $\mathrm{Cu}$ chains: Only one chain is affected for in-chain doping by $\mathrm{Zn}$ instead of three chains in the case of out-of-chain Sidoping [42]. This has provided Pouget et al. 44 with a simple model explaining the factor 3 , between $\mathrm{Si}$ and $\mathrm{Zn}$ doping, in the respective decrease of $T_{S P}$ and in the critical concentration above which the SP LRO is destroyed. According to the present study, we can generalize this last 
result concerning $\mathrm{Zn}$ to the other $\mathrm{Cu}$ substituents, $\mathrm{Ni}$ and $\mathrm{Mg}$, which produce smaller local distortions. As concerns the Mn impurity, which leads to stronger distortions than $\mathrm{Zn}$, the fact that $T_{S P}$ has the same concentration dependence tends to show that Mn-doping affects only one $\mathrm{Cu}$ chain. Note that the furthest analyzed atomic distance probed by our EXAFS experiment is shorter than that to the next $\mathrm{Cu}$ on a neighboring chain $(4.25 \AA)$. In summary, the universality of the SP transition seems to be related to the transverse weakening of the dimerization which depends therefore essentially on the position of the impurity, outside or inside the chains.

Lastly, let us come back to the difference in the distortions induced by different concentrations of the same impurity. The Ni-O $(2)$ distance to the first neighbors increases more for $6 \% \mathrm{Ni}$ than for $2 \% \mathrm{Ni}$ doping with respect to the $\mathrm{Cu}$ environment. The higher doped compound, also, has a more disordered environment. It is interesting to note that these concentrations lie before and after the end of the $T_{S P}$ line, and on each side of the transition from dimerized to uniform AF LRO. This result underlines some influence of the disorder in the disappearance of the spin-Peierls phase.

\section{Conclusion}

In the present study, the EXAFS technique was used as a powerful probe of the local atomic arrangement around various impurities in the paramagnetic state of doped $\mathrm{CuGeO}$ This technique has allowed us to quantify the distortions in the impurity environment, such as the modification of the shape of the $\mathrm{O}$ octahedron around the $\mathrm{Cu}$ site, and to evaluate the local modification of the angles and distances relevant for the magnetic properties. An increase of the first neighbor distance to the $\mathrm{Cu}$ site was observed when substituting $\mathrm{Cu}$ by $\mathrm{Ni}, \mathrm{Zn}$ and $\mathrm{Mn}$, in this order. An elongation of the distances to the further neighbors was evidenced in the case of Mn-doping which thus produces the largest alteration among the $\mathrm{Cu}$ site impurities. The angle $\alpha$ of the $\mathrm{Cu}-\mathrm{O}-\mathrm{Cu}$ superexchange path leading to the AF $J_{1}$ interaction between $\mathrm{Cu}^{2+}$ first neighbors was found to decrease progressively when doping with $\mathrm{Ni}$, then with $\mathrm{Zn}$ or $\mathrm{Mn}$, and at last with $\mathrm{Si}$. These results have allowed establishing the local weakening of the $J_{1}$ interaction for $\mathrm{Ni} / \mathrm{Cu}$ substitution, its almost cancellation in the case of $\mathrm{Mn} / \mathrm{Cu}$ substitution and, lastly, its change of sign for $\mathrm{Si}$ doping on the Ge site, leading to a small ferromagnetic coupling between each set of $\mathrm{Cu}$ pairs of two adjacent chains.

In a global scheme where each impurity is considered to release magnetic solitonic defects, an analysis of the structural distortions of the doped $\mathrm{CuGeO}_{3}$ in relation with their magnetic properties could then be proposed. A good description of the magnetic behavior of Si-doped compounds is obtained calling upon ferromagnetic pairs of spins $1 / 2$, which yields in particular a simple explanation for the renormalization of $T_{N}$ for $\mathrm{Si}$ with respect to Zn-doping. Another interesting consequence of this study concerns the $\mathrm{Cu}$-site impurities for which the amplitude of the structural distortion, rather than the magnetic moment of the impurity, is found to be correlated to the variation of $T_{N}$. This last result underlines the importance of the structural distortions in the magnetic behavior of spin-Peierls doped compounds and opens the way to models taking into account realistic structural parameters.

We would like to thank B. Canals, P. Monod, R. Mélin, D. Khomskii and N. Laflorencie for fruitful discussions.

\section{References}

1. M. Hase, I. Terasaki, K. Uchinokura, Phys. Rev. Lett. 70, 3651 (1993)

2. J.-P. Pouget, L.-P. Regnault, M. Ä̈n, B. Hennion, J.-P. Renard, P. Veillet, G. Dhalenne, A. Revcolevschi, Phys. Rev. Lett. 72, 4037 (1994)

3. M. Hase, I. Terasaki, Y. Sasago, K. Uchinokura, H. Obara, Phys. Rev. Lett. 71, 4059 (1993); S. B. Oseroff, S.-W. Cheong, B. Aktas, M. F. Hundley, Z. Fisk, L. W. Rupp, Jr., Phys. Rev. Lett. 74, 1450 (1995); Y. Ajiro, T. Asano, F. Masui, M. Mekata, H. Aruga-Katori, T. Goto, H. Kikuchi, Phys. Rev. B 51, 9399 (1995)

4. S. Coad, J. G. Lussier, D. F. McMorrow, D. McK. Paul, J. Phys.: Condens. Matter 8, 6251 (1996)

5. B. Grenier, J.-P. Renard, P. Veillet, C. Paulsen, G. Dhalenne, A. Revcolevschi, Phys. Rev. B 58, 8202 (1998)

6. J. -P. Renard, K. Le Dang, P. Veillet, G. Dhalenne, A. Revcolevschi, L.-P. Regnault, Europhys. Lett. 30, 475 (1995)

7. B. Grenier, J.-P. Renard, P. Veillet, C. Paulsen, R. Calemczuk, G. Dhalenne, A. Revcolevschi, Phys. Rev. B 57, 3444 (1998)

8. B. Grenier, P. Monod et al., in preparation

9. S. Katano, O. Fujita, J. Akimitsu, M. Nishi, K. Kakurai, Y. Fujii, Phys. Rev. B 57, 10280 (1998)

10. M. Hiroi, T. Hamamoto, M. Sera, H. Nojiri, N. Kobayashi, M. Motokawa, O. Fujita, A. Ogiwara, J. Akimitsu, Phys. Rev. B 55, R6125 (1997)

11. Y. J. Wang, Y. J. Kim, R. J. Christianson, S. C. LaMarra, F. C. Chou, T. Masuda, I. Tsukada, K. Uchinokura R. J. Birgeneau, J. Phys. Soc. Japan 72, 1544 (2003)

12. T. Masuda, A. Fujioka, Y. Uchiyama, I. Tsukada, K. Uchinokura, Phys. Rev. Lett. 80, 4566 (1998); T. Masuda, I. Tsukada, K. Uchinokura, Y. J. Wang, V. Kiryukhin, and R. J. Birgeneau, Phys. Rev. B 61, 4103 (2000); T. Masuda, N. Koide, K. Uchinokura, Progress Theor. Phys. Suppl. 145, 306 (2002)

13. B. Grenier, P. Monod, M. Hagiwara, M. Matsuda, K. Katsumata, S. Clément, J.-P. Renard, A. L. Barra, G. Dhalenne, A. Revcolevschi, Phys. Rev. B 65, 94425 (2002)

14. G. Castilla, S. Chakravarty, V. J. Emery, Phys. Rev. Lett. 75, 1823 (1995); J. Riera and A. Dobry, Phys. Rev. B 51, 16098 (1995); K. Fabricius, A. Klümper, U. Löw, B. Büchner, T. Lorenz, G. Dhalenne, and A. Revcolevschi, Phys. Rev. B 57, 1102 (1998)

15. M. Nishi, O. Fujita, J. Akimitsu, Phys. Rev. B 50, 6508 (1994); L. P. Regnault, M. Ä̈n, B. Hennion, G. Dhalenne, A. Revcolevschi, Phys. Rev. B 53, 5579 (1996)

16. J. B. Goodenough, in Magnetism and the chemical bond (John Wiley and Sons, New York, 1963). 
17. M. Braden, G. Wilkendorf, J. Lorenzana, M. Ä̈n, G. J. McIntyre, M. Behruzi, G. Heger, G. Dhalenne, A. Revcolevschi, Phys. Rev. B 54, 1105 (1996)

18. W. Geertsma and D. Khomskii, Phys. Rev. B 54, 3011 (1996).

19. M. Newville, P. Livins, Y. Yacoby, J. J. Rehr, E. A. Stern, Phys. Rev. B 47, 14126 (1993) ; S. I. Zabinsky, J. J. Rehr, A. Ankudinov, R. C. Albers, M. J. Eller, Phys. Rev. B 52, 2995 (1995) ; M. Newville, R. Ravel, D. Haskel, J. J. Rehr, E. A. Stern, Y. Yacoby, Physica B 208-209, 154 (1995)

20. H. Völlenkle, A. Wittmann, H. Nowotny, Monatsh. Chem. 98, 1352 (1967)

21. M. Braden, E. Ressouche, B. Büchner, R. Keßler, G. Heger, G. Dhalenne, A. Revcolevschi, Phys. Rev. B 57, 11497 (1998)

22. R. D. Shannon, Acta Cryst. A 32, 751 (1976)

23. H. H. Otto, M. Meibohm, Z. Kristallogr. 214, 558 (1999)

24. M. Baenitz, C. Geibel, M. Dischner, G. Sparn, F. Steglich, H. H. Otto, M. Meibohm, A. A. Gippius, Phys. Rev. B 62 , $12201(2000)$

25. M. Weiden, R. Hauptmann, W. Richter, C. Geibel, P. Hellmann, M. Köppen, F. Steglich, M. Fischer, P. Lemmens, G. Güntherodt, A. Krimmel, G. Nieva, Phys. Rev. B 55, 15067 (1997)

26. H. Fukuyama, T. Tanimoto, M. Saito, J. Phys. Soc. Jpn. 65, $1182(1996)$

27. M. Fabrizio, R. Mélin, Phys. Rev. Lett. 78, 3382 (1997); M. Fabrizio, R. Mélin, Phys. Rev. B 56, 5996 (1997);M. Fabrizio, R. Mélin, J. Souletie, Eur. Phys. J. B 10, 607 (1999)

28. D. Khomskii, W. Geertsma, M. Mostovoy, Czech. J. Phys. 46, Suppl. S6, 3240 (1996); M. Mostovoy, D. Khomskii, Z. Phys. B 103, 209 (1997)

29. M. Mostovoy, D. Khomskii, J. Knoester, Phys. Rev. B 58, 8190 (1998)

30. E. Sorensen, I. Affleck, D. Augier, D. Poilblanc, Phys. Rev. B 58, R14701 (1998); A. Dobry, P. Hansen, J. Riera, D. Augier, D. Poilblanc, Phys. Rev. B 60, 4065 (1999); D. Augier, J. Riera, D. Poilblanc, Phys. Rev. B 61, 6741 (2000)

31. N. Laflorencie, D. Poilblanc, Phys. Rev. Lett. 90, 157202 (2003); N. Laflorencie, D. Poilblanc, A. W. Sandvik, Phys. Rev. B 69, 212412 (2004)

32. L.-P. Regnault, J.-P. Renard, G. Dhalenne, A. Revcolevschi, Europhys. Lett. 32, 579 (1995)

33. Y. Sasago, N. Koide, K. Uchinokura, M.C. Martin, M. Hase, K. Hirota, G. Shirane, Phys. Rev. B54, R6835 (1996)

34. B. Grenier, L.-P. Regnault et al., in preparation

35. K. Manabe, H. Ishimoto, N. Koide, Y. Sasago, K. Uchinokura, Phys. Rev. B 58, R575 (1998)

36. K. M. Kojima, Y. Fudamoto, M. Larkin, G. M. Luke, J. Merrin, B. Nachumi, Y. J. Uemura, M. Hase, Y. Sagaso, K. Uchinokura, Y. Ajiro, A. Revcolevschi, J.-P. Renard, Phys. Rev. Lett. 79, 503 (1997)

37. B. Büchner, U. Ammerahl, T. Lorenz, W. Brenig, G. Dhalenne, A. Revcolevschi, Phys. Rev. Lett. 77, 1624 (1996)

38. W. Geertsma, D. Khomskii, cond-mat/0007421

39. R. Werner, C. Gros, M. Braden, Phys. Rev. B 59, 14356 (1999)

40. H. Wolfram, H. H. Otto, M. Cwik, M. Braden, G. André, F. Bourée, M. Baenitz, F. Steglich, Phys. rev. B 69, 144115 (2004)
41. B. Grenier, L.-P. Regnault, J.E. Lorenzo, J. Voiron, J. Bossy, J.-P. Renard, G. Dhalenne, A. Revcolevschi, Europhys. Lett. 44, 511 (1998)

42. J.-P. Pouget, S. Ravy, J.-P. Schoeffel, G. Dhalenne, A. Revcolevschi, Eur. Phys. J. B 38, 581 (2004)

43. B. Grenier, L.-P. Regnault, J.E. Lorenzo, J.-P. Renard, G. Dhalenne, A. Revcolevschi, Physica B 280, 313 (2000)

44. J.-P. Schoeffel, J.-P. Pouget, G. Dhalenne, A. Revcolevschi, Phys. Rev. B 53, 14971 (1996) 